\title{
Use of multilocus variable-number tandem repeat analysis in molecular subtyping of Salmonella enterica serovar Typhi isolates
}

\section{Correspondence \\ Chien-Shun Chiou \\ nipmcsc@cdc.gov.tw}

Received 16 August 2011

Accepted 7 October 2011

\section{Yung-Yen Tien, ${ }^{1}$ Hiroshi Ushijima, ${ }^{2}$ Masashi Mizuguchi, ${ }^{3}$ Shiu-Yun Liang ${ }^{4}$ and Chien-Shun Chiou ${ }^{4,5}$}

\author{
${ }^{1}$ Department of International Trade, Tamkang University, New Taipei, Taiwan, ROC \\ ${ }^{2}$ Aino Health Science Center, Aino University, Tokyo, Japan \\ ${ }^{3}$ Institute of International Health, Graduate School of Medicine, University of Tokyo, Tokyo, Japan \\ ${ }^{4}$ The Central Region Laboratory, Center for Research and Diagnostics, Centers for Disease Control, \\ Taichung, Taiwan, ROC \\ ${ }^{5}$ Institute of Medicine, Chung Shan Medical University, Taichung, Taiwan, ROC
}

\begin{abstract}
We evaluated 11 variable number tandem repeat (VNTR) markers for the epidemiological investigation of Salmonella enterica serovar Typhi (S. Typhi) infection and compared the results to those obtained by PFGE. PFGE, using one or two restriction enzymes (Xbal and B/nl), was insufficient to differentiate between some isolates that were epidemiologically unlinked. Multilocus variable-number tandem repeat analysis (MLVA)-8, based on analysis of the eight most variable VNTRs, displayed a high level of discrimination when distinguishing between epidemiologically unlinked isolates that could not be discerned by PFGE with two enzymes. An MLVA-8 typing scheme could be implemented as a routine subtyping tool for the epidemiological investigation of $S$. Typhi infections. Because seven of the 11 VNTRs are highly variable, the VNTR markers may only be useful in determining genetic relationships among very closely related isolates in short-term epidemiological studies and not for discerning S. Typhi clones.
\end{abstract}

\section{INTRODUCTION}

Salmonella enterica subspecies enterica serovar Typhi $(S$. Typhi) causes typhoid fever, a disease transmitted primarily through the faecal-oral route. Typhoid fever is most prevalent in South-Central and South-East Asia, causing an estimated 22 million cases and 0.22 million deaths in the year 2000 (Crump et al., 2004). In developed countries, the incidence of typhoid fever has declined markedly and it is now predominantly associated with travellers returning from endemic areas (Connor \& Schwartz, 2005).

Characterization by various genotyping methods can provide useful information when trying to assess genetic connections among isolates for short- and long-term epidemiological investigations (Spratt, 2004). Highly discriminatory typing methods for the differentiation of closely related isolates, such as those derived from a very recent common ancestor over a period of months or years, are suitable for the investigation of short-term epidemiology,

Abbreviations: $\mathrm{Cl}$, confidence interval; DI, discriminatory index; MLVA multilocus variable-number tandem repeat analysis; MST, minimum spanning tree; TR, tandem repeat; VNTR, variable number tandem repeat. such as disease outbreaks and disease surveillance (HyytiäTrees et al., 2007). Typing methods, such as multilocus sequence typing (MLST) (Maiden et al., 1998), that can distinguish between clones among isolates evolved over a period of decades are appropriate for long-term epidemiological studies, for example the global spread of hypervirulent clones of rapidly evolving Neisseria meningitidis (Caugant et al., 1986; Chiou et al., 2006). MLST and single nucleotide polymorphism analysis have been used for evolutionary and global epidemiology studies of $S$. Typhi (Kidgell et al., 2002; Octavia \& Lan, 2007; Roumagnac et al., 2006). PFGE is a highly discriminatory method for the differentiation of most bacterial pathogens. It has been widely used as a subtyping tool for the investigation of disease outbreaks (Hyytiä-Trees et al., 2007) and it was also adopted as a standard subtyping tool for an international foodborne disease surveillance network (Swaminathan et al., 2006). However, in some cases, PFGE is insufficient in distinguishing between epidemiologically unlinked isolates of genetically monomorphic pathogens, such as Shigella sonnei (Liang et al., 2007), Escherichia coli O157: H7 (Noller et al., 2003), Salmonella enterica serovar Enteritidis (Boxrud et al., 2007) and Salmonella enterica serovar Typhimurium (Chiou et al., 2010; Torpdahl et al., 2007). On the other 
hand, PFGE is too discriminatory to be an appropriate typing tool for the investigation of the global spread of bacterial clones (Spratt, 2004).

Multilocus variable-number tandem repeat analysis (MLVA) is a well-known typing method that has been successfully developed for many bacterial species or for serovars within a species (Liang et al., 2007; Liao et al., 2006; Lindstedt et al., 2003; Marsh et al., 2006; Noller et al., 2003). Based on the analysis of multilocus variable-number tandem repeats (VNTRs), MLVA can be an extremely powerful method for distinguishing between closely related isolates in the investigation of disease outbreaks (Liang et al., 2007; Noller et al., 2003; Torpdahl et al., 2007).

$S$. Typhi is a genetically monomorphic bacterial pathogen (Kidgell et al., 2002). In our experience, PFGE is not discriminatory enough, in some cases, to distinguish between epidemiologically unlinked $S$. Typhi isolates. In the present study, we screened the released genomic sequences of Salmonella enterica serovars Typhi and Paratyphi A for VNTRs. We assessed the diversity of the VNTRs and compared the discriminatory power of MLVA, based on various combinations of VNTRs, against that of PFGE. Finally, we investigated the usefulness of MLVA data in the epidemiological study of $S$. Typhi infection.

\section{METHODS}

Bacterial isolates. Bacterial isolates were collected in the Central Region Laboratory, Center for Research and Diagnostics, Centers for Disease Control, Taiwan. In total, $125 \mathrm{~S}$. Typhi isolates were collected between 1996 and 2009 (Table 1). Of the 125 S. Typhi isolates, 38 were recovered from patients returning from other countries, including Bangladesh (1 isolate), Cambodia (9), China (3), France (1), India (2), Indonesia (18), Myanmar (2), Philippines (1) and Thailand (1). Isolates that had no epidemiological record identifying country of origin were considered as having originated in Taiwan.

PFGE. The PulseNet PFGE protocol for Salmonella and other enterobacteria (Ribot et al., 2006) was used for PFGE analysis, except that a smaller amount of $\mathrm{Xba \textrm {I }}(10 \mathrm{U})$ and $B \ln \mathrm{I}(16 \mathrm{U})$ was used for the digestion of each slice.

Identification of tandem repeat loci with VNTR potential. $S$. Typhi and S. Paratyphi A are more closely related at the DNA level than other Salmonella serovars, as indicated by whole genome sequence comparisons (McClelland et al., 2004). The genomic sequences of $S$. Typhi strain CT18 (GenBank accession no. AL513382), S. Typhi strain Ty2 (accession no. AE014613), and S. Paratyphi A strain ATCC9150 (accession no. CP000026) were available at the beginning of this study. To explore as many VNTRs as possible in the development of an MLVA method for S. Typhi, the genomic sequence of $S$. Paratyphi A strain ATCC9150 was included in the in silico searches using the VNTRDB computer software (Chang et al., 2007). Tandem repeat (TR) sequences that were polymorphic among the three genomes, had $>90 \%$ sequence similarity and had no indels within the repeat sequence array were selected and evaluated with 11 distantly related $S$. Typhi isolates. The selected TRs that were easily amplified by PCR and had only one amplicon in the isolates tested were further examined for allelic diversity among the $125 \mathrm{~S}$. Typhi isolates.
PCR. To perform the PCR, primers for the TRs were designed using the free Primer3 program (http://frodo.wi.mit.edu/primer3/). PCRs were carried out using a GeneAmp PCR System 9600 thermocycler (Applied Biosystems). For amplification by PCR, crude bacterial DNA was prepared using a boiling method as described previously (Liang et al., 2007). Each $10 \mu \mathrm{l}$ PCR mixture contained $1 \times$ PCR buffer, $1 \%$ DMSO, $3 \mathrm{mM} \mathrm{MgCl} 2,25-100 \mathrm{nM}$ each primer, $200 \mu \mathrm{M}$ each deoxyribonucleotide, $1 \mathrm{U}$ recombinant SuperNew Taq DNA polymerase (Jier Sheng Company) and $1 \mu$ DNA template prepared by the boiling method. PCR was performed with an initial denaturing step at $94{ }^{\circ} \mathrm{C}$ for $5 \mathrm{~min}$, followed by 30 cycles of amplification at $94{ }^{\circ} \mathrm{C}$ for $30 \mathrm{~s}, 55{ }^{\circ} \mathrm{C}$ for $60 \mathrm{~s}$ and $72{ }^{\circ} \mathrm{C}$ for $30 \mathrm{~s}$, and an extension step at $72{ }^{\circ} \mathrm{C}$ for $5 \mathrm{~min}$. Occasionally, for some loci, no DNA product was amplified in the multiplex PCR. In these cases, amplification was performed again in a singleplex PCR. For strains where certain TR loci were not amplified when using genomic DNA prepared by the boiling method, genomic DNA was extracted using a commercial kit (GeneAid). The primer sequences for the 11 identified VNTRs are listed in Table 2.

Analysis of PCR amplicons. PCR products were separated by capillary electrophoresis on an ABI Prism 3130XL Genetic Analyzer (Applied Biosystems). The sizes of the amplicons were determined using GeneScan data analysis software version 3.7 (Applied Biosystems) based on the GeneScan 500 LIZ size standard markers. All amplicons with different sizes from each locus were subjected to nucleotide sequence analysis to verify the repeat sequence and the number of repeat units within the amplicons.

Data analysis. PFGE images were analysed using the fingerprint analysis software BioNumerics version 4.5 (Applied Maths). A PFGE genotype was defined as a PFGE pattern with one or more DNA bands different from the others. The number of repeat units for each allele was converted from the amplicon sizes and saved as a 'character type' in the BioNumerics database. An allele that contained a deletion close to a repeat region at Sty20 was assigned to a character type based on the fragment size (bp). The VNTR profiles were subjected to cluster analysis using the minimum spanning tree (MST) and categorical coefficient algorithms provided in the BioNumerics software. The creation of hypothetical types (missing links) was permitted in order to introduce hypothetical types, the group minimum size was set at 2 and the maximum neighbour distance was set at 1 . To compare the discriminatory power of PFGE and MLVA based on combinations of various numbers of VNTRs, the discrimination index (DI) (using Simpson's index of diversity) and $95 \%$ confidence interval (CI) were calculated using formulas as described previously (Grundmann et al., 2001; Hunter, 1990). The degree of polymorphism for each VNTR was indicated by Nei's diversity index, calculated as $1-\sum$ (allelic frequency $)^{2}$.

\section{RESULTS AND DISCUSSION}

\section{VNTRs and allelic diversity}

To explore as many VNTRs as possible, we used the computer program VNTRDB (Chang et al., 2007) to explore TRs with VNTR potential from the genomic sequences of two $S$. Typhi and one $S$. Paratyphi A strains. Forty-five TRs that had $>90 \%$ repeat sequence similarity and no indel site within the repeat region were selected for preliminary evaluation. The primers for the 45 TRs were examined using a panel of 11 distantly related $S$. Typhi isolates. The primer screening process selected 34 TRs that were easily amplified by PCR and had only one amplicon in the 
Table 1. Salmonella enterica serovar Typhi isolates used in this study

\begin{tabular}{|c|c|c|c|c|c|c|}
\hline $04-783550$ & $2009 / 1 / 25$ & X.129 & A. 01 & M.125 & $\mathrm{C} 1$ & Indonesia \\
\hline ST10867 & $1998 / 6 / 11$ & X.001 & A. 01 & M.089 & $\mathrm{C} 2$ & Indonesia \\
\hline ST15788 & $1999 / 6 / 28$ & Х.001 & A. 01 & M.076 & $\mathrm{C} 2$ & \\
\hline S05.0884 & $2005 / 5 / 4$ & X.001 & A. 01 & M.122 & $\mathrm{C} 2$ & Indonesia \\
\hline C06.1398 & $2006 / 7 / 31$ & X.001 & A. 01 & M.065 & C2 & Indonesia \\
\hline N04.0024 & $2003 / 12 / 27$ & X.041 & A.12 & M.124 & C3 & Indonesia \\
\hline N04.0030 & $2004 / 9 / 3$ & X.041 & A. 12 & M.036 & C3 & Indonesia \\
\hline $04-480280$ & $2009 / 5 / 8$ & X.041 & A. 12 & M.126 & $\mathrm{C} 3$ & Indonesia \\
\hline $04-925983$ & $2009 / 5 / 10$ & X.136 & A.71 & M.053 & C4/Epi-1 & \\
\hline C05.0088 & $2005 / 1 / 14$ & X.016 & A. 15 & M.084 & C6 & \\
\hline N05.0022 & $2005 / 7 / 30$ & X.016 & A. 15 & M.112 & C6 & \\
\hline ST18659 & $2000 / 2 / 7$ & X.016 & A.14 & M.026 & C7 & \\
\hline N04.0594 & $2004 / 2 / 13$ & Х.016 & A. 14 & M.130 & C7 & \\
\hline 04-139944 & $2008 / 9 / 18$ & X.106 & A. 15 & M.146 & C8 & \\
\hline 04-750709 & $2009 / 5 / 2$ & X.106 & A. 15 & M.147 & $\mathrm{C} 8$ & \\
\hline ST11628 & $1998 / 9 / 7$ & X.022 & A. 19 & M.151 & C9 & \\
\hline N04.0025 & $2004 / 1 / 3$ & X.022 & A. 19 & M.157 & C9 & \\
\hline NK07.052 & $2007 / 8 / 15$ & X.022 & A. 19 & M.040 & C9 & \\
\hline NB07.038 & $2007 / 8 / 20$ & X.022 & A. 19 & M.153 & C9 & \\
\hline CG07.254 & $2007 / 9 / 29$ & X.022 & A. 19 & M.155 & C9 & \\
\hline 04-139907 & $2008 / 5 / 19$ & X.022 & A. 19 & M.040 & C9 & \\
\hline C04.0342 & $2004 / 2 / 5$ & X.007 & A. 04 & M.023 & C11 & \\
\hline N04.0028 & $2004 / 10 / 20$ & X.007 & A. 04 & M.021 & $\mathrm{C} 11$ & \\
\hline NA07.528 & $2007 / 11 / 26$ & X.007 & A. 04 & M.068 & C11 & \\
\hline $04-764625$ & $2009 / 5 / 13$ & X.007 & A. 04 & M.073 & C11 & \\
\hline S04.2684 & $2004 / 7 / 17$ & X.057 & A.11 & M.096 & C12/Epi-3 & \\
\hline S04.2685 & $2004 / 7 / 17$ & X.057 & A. 11 & M.097 & C12/Epi-3 & \\
\hline S04.2768 & $2004 / 7 / 22$ & Х.057 & A.11 & M.029 & C12/Epi-3 & \\
\hline N05.0009 & $2005 / 6 / 24$ & X.067 & A. 03 & M.133 & C13/Epi-4 & \\
\hline N05.0011 & $2005 / 6 / 29$ & X.067 & A. 03 & M.038 & C13/Epi-4 & \\
\hline N05.0018 & $2005 / 7 / 14$ & X.067 & A. 03 & M.004 & C13/Epi-4 & \\
\hline N05.0021 & $2005 / 7 / 18$ & X.067 & A. 03 & M.038 & C13/Epi-4 & \\
\hline N05.0008 & $2005 / 6 / 19$ & X.064 & A. 02 & M.004 & C14/Epi-4 & \\
\hline N05.0010 & $2005 / 6 / 27$ & X.064 & A. 02 & M.004 & C14/Epi-4 & \\
\hline N05.0015 & $2005 / 6 / 29$ & X.064 & A. 02 & M.004 & C14/Epi-4 & \\
\hline N05.0013 & $2005 / 6 / 30$ & X.064 & A. 02 & M.005 & C14/Epi-4 & \\
\hline N05.0016 & $2005 / 7 / 7$ & X.064 & A. 02 & M.005 & C14/Epi-4 & \\
\hline N05.0019 & $2005 / 7 / 20$ & X.064 & A. 02 & M.004 & C14/Epi-4 & \\
\hline N05.0020 & $2005 / 7 / 21$ & X.064 & A. 02 & M.005 & C14/Epi-4 & \\
\hline N05.0007 & $2005 / 6 / 21$ & X.065 & A. 34 & M.005 & Epi-4 & \\
\hline N05.0012 & $2005 / 6 / 30$ & X.063 & A. 10 & M.131 & Epi-4 & \\
\hline N05.0017 & $2005 / 7 / 15$ & X.066 & A. 33 & M.005 & Epi-4 & \\
\hline
\end{tabular}


Table 1. cont.

\begin{tabular}{|c|c|c|c|c|c|c|}
\hline Isolate ID & Isolation date & PFGE-XbaI & PFGE-BlnI & MLVA type* & $\begin{array}{l}\text { PFGE cluster/ } \\
\text { Epi cluster }\end{array}$ & Country of origin \\
\hline N04.0040 & $2004 / 3 / 13$ & X.035 & A. 66 & M.011 & C15/Epi-5 & Cambodia \\
\hline N04.0042 & $2004 / 4 / 13$ & X.035 & A. 66 & M.011 & C15/Epi-5 & Cambodia \\
\hline N04.0010 & $2004 / 4 / 15$ & X.035 & A. 66 & M.011 & C15/Epi-5 & Cambodia \\
\hline N04.0007 & $2004 / 3 / 20$ & X.035 & A. 64 & M.050 & Epi-5 & Cambodia \\
\hline NM04.078 & $2001 / 5 / 6$ & X.037 & A. 56 & M.175 & C16 & \\
\hline N04.0033 & $2004 / 9 / 16$ & X.037 & A. 56 & M.216 & $\mathrm{C} 16$ & Myanmar \\
\hline ST4256 & $1996 / 10 / 14$ & X.008 & A. 08 & M.094 & & \\
\hline ST7418 & $1997 / 6 / 27$ & X.014 & A. 26 & M.082 & & \\
\hline ST8856 & $1997 / 10 / 30$ & X.005 & A. 40 & M.139 & & \\
\hline ST9093 & $1997 / 11 / 29$ & X.033 & A. 62 & M.249 & & \\
\hline ST9148 & $1997 / 12 / 13$ & X.022 & A. 20 & M.154 & & \\
\hline ST10070 & $1998 / 3 / 8$ & X.031 & A. 32 & M.181 & & India \\
\hline ST10136 & $1998 / 3 / 14$ & X.010 & A. 46 & M.149 & & China \\
\hline ST11052 & 1998/7/1 & X.036 & A. 67 & M.083 & & Cambodia \\
\hline ST11786 & $1998 / 10 / 5$ & X.034 & A. 61 & M.092 & & Cambodia \\
\hline ST14115 & $1998 / 12 / 10$ & X.006 & A. 41 & M.138 & & Indonesia \\
\hline ST14969 & $1998 / 12 / 30$ & X.011 & A. 24 & M.176 & & \\
\hline ST15395 & $1999 / 3 / 21$ & X.009 & A. 06 & M.061 & & France \\
\hline ST18361 & $1999 / 12 / 6$ & X.180 & A. 21 & M.165 & & \\
\hline ST18600 & $2000 / 1 / 20$ & Х.040 & A. 42 & M.243 & & Thailand \\
\hline ST20564 & $2000 / 9 / 11$ & X.030 & A. 44 & M.252 & & \\
\hline ST20567 & $2000 / 9 / 20$ & X.022 & A. 23 & M.156 & & Indonesia \\
\hline ST20608 & $2000 / 9 / 24$ & X.003 & A. 24 & M.180 & & Indonesia \\
\hline ST20882 & $2000 / 11 / 22$ & X.004 & A. 31 & M.136 & & Indonesia \\
\hline ST21149 & $2001 / 1 / 29$ & X.013 & A. 45 & M.244 & & China \\
\hline ST21562 & $2001 / 3 / 28$ & X.025 & A. 35 & M.072 & & \\
\hline ST22061 & $2001 / 5 / 21$ & X.015 & A. 25 & M.081 & & Indonesia \\
\hline ST28481 & $2002 / 7 / 13$ & X.186 & A. 76 & M.150 & & \\
\hline ST30227 & $2002 / 11 / 15$ & X.020 & A. 37 & M.090 & & \\
\hline NM04.016 & $2003 / 7 / 12$ & X.042 & A. 57 & M.224 & & \\
\hline ST33416 & $2003 / 10 / 23$ & X.037 & A. 59 & M.183 & & \\
\hline ST33681 & $2003 / 11 / 26$ & X.017 & A. 43 & M.098 & & \\
\hline N04.0004 & $2003 / 12 / 31$ & X.038 & A. 51 & M.135 & & \\
\hline S04.0039 & $2004 / 1 / 2$ & X.061 & A. 55 & M.247 & & \\
\hline N04.0087 & $2004 / 1 / 7$ & X.039 & A. 52 & M.058 & & Philippines \\
\hline N04.0187 & $2004 / 1 / 13$ & X.021 & A. 16 & M.025 & & \\
\hline N04.0265 & $2004 / 1 / 14$ & X.001 & A. 38 & M.080 & & \\
\hline S04.0152 & $2004 / 1 / 14$ & X.062 & A. 09 & M.128 & & Indonesia \\
\hline C04.0728 & $2004 / 4 / 23$ & X.026 & A. 28 & M.250 & & \\
\hline N04.0034 & $2004 / 5 / 6$ & X.045 & A. 65 & M.234 & & Cambodia \\
\hline N04.0019 & $2004 / 5 / 17$ & X.052 & A. 54 & M.145 & & Bangladesh \\
\hline S04.1788 & $2004 / 5 / 19$ & X.045 & A. 61 & M.047 & & Cambodia \\
\hline C04.0905 & $2004 / 5 / 24$ & X.045 & A. 64 & M.232 & & \\
\hline N04.0018 & $2004 / 6 / 27$ & X.046 & A. 61 & M.233 & & Cambodia \\
\hline S04.2422 & $2004 / 7 / 1$ & X.043 & A. 22 & M.046 & & \\
\hline N04.0031 & $2004 / 9 / 20$ & X.053 & A. 01 & M.134 & & \\
\hline NK04.080 & $2004 / 10 / 10$ & X.047 & A. 07 & M.069 & & \\
\hline N04.0027 & $2004 / 11 / 6$ & X.056 & A. 50 & M.101 & & \\
\hline N04.0026 & $2004 / 11 / 9$ & X.050 & A. 49 & M.095 & & \\
\hline S05.1491 & $2005 / 7 / 4$ & X.055 & A. 30 & M.093 & & \\
\hline C05.1536 & $2005 / 8 / 2$ & X.012 & A. 24 & M.043 & & \\
\hline C05.1632 & $2005 / 8 / 10$ & X.068 & A. 27 & M.115 & & \\
\hline EA05.036 & $2005 / 8 / 21$ & X.054 & A. 47 & M.109 & & \\
\hline CG06.001 & $2006 / 1 / 3$ & X.071 & A. 35 & M.071 & & \\
\hline CA06.017 & $2006 / 3 / 8$ & X.072 & A.18 & M.172 & & \\
\hline
\end{tabular}


Table 1. cont.

\begin{tabular}{|c|c|c|c|c|c|c|}
\hline Isolate ID & Isolation date & PFGE-XbaI & PFGE-BlnI & MLVA type & $\begin{array}{l}\text { PFGE cluster/ } \\
\text { Epi cluster }\end{array}$ & Country of origin \\
\hline C06.0890 & $2006 / 4 / 20$ & X.073 & A. 05 & M.132 & & \\
\hline NK06.018 & $2006 / 4 / 25$ & Х.074 & A. 39 & M.246 & & \\
\hline C06.1067 & $2006 / 5 / 29$ & X.076 & A. 29 & M.102 & & China \\
\hline SB06.100 & $2006 / 10 / 9$ & X.037 & A. 60 & M.226 & & India \\
\hline C07.0093 & $2007 / 1 / 22$ & X.081 & A. 36 & M.110 & & \\
\hline ND07.001 & $2007 / 6 / 18$ & X.085 & A. 53 & M.070 & & \\
\hline SB07.062 & $2007 / 8 / 31$ & Х.037 & A.73 & M.106 & & \\
\hline $04-417166$ & $2008 / 5 / 28$ & X.103 & A. 69 & M.016 & & Myanmar \\
\hline $04-347232$ & $2008 / 5 / 31$ & X.078 & A. 74 & M.075 & & \\
\hline 04-820220 & $2008 / 7 / 30$ & X.012 & A.72 & M.179 & & Indonesia \\
\hline
\end{tabular}

${ }^{*}$ MLVA type was designated using profiles of 11 VNTRs.

isolates tested. The 34 TRs were further examined for allelic diversity in the $125 \mathrm{~S}$. Typhi isolates. Only 12 of the 34 TRs displayed polymorphisms in the $125 \mathrm{~S}$. Typhi isolates tested. Ten of the 12 VNTRs had been described previously, including TR1 (Sty41), TR2 (Sty45) and TR5 (Sty42) (Liu et al., 2003); STTR5 (Sty44) (Lindstedt et al., 2003), Sal02 (Sty37), Sal06 (Sty39), Sal20 (Sty40) and Sal10 (Sty43) (Ramisse et al., 2004); and TR4500 (Sty20) and TR4699 (Sty25) (Octavia \& Lan, 2009). Two novel VNTRs, Sty2 and Sty3, were discovered in the present study. Sty37 and Sty 44 are also variable in S. Paratyphi A (Tien et al., 2011). Sty43 (Sal10) had two alleles identified in the 125 isolates; one of the alleles contained the $739 \mathrm{bp}$ insertion sequence element IS200 located immediately upstream of the repeat region. This VNTR was excluded from the data analysis because of its extremely low diversity and the difficulty with which it was analysed by capillary electrophoresis on an ABI Prism 3130XL Genetic Analyzer.

Table 2 lists the characteristics of the 11 VNTRs. The sizes of the repeat units for the 11 VNTRs ranged from 3 to $44 \mathrm{bp}$. Depending on the locus, the number of alleles varied from 2 to 38 in the 125 isolates characterized, and the alleles contained from 1 up to as many as 43 repeats. Seven VNTRs (Sty20, Sty25, Sty37, Sty40, Sty41, Sty44 and Sty45) were highly diverse, with diversity values of 0.70 0.95. Diversity at a VNTR marker is a function of both the number of alleles and their distribution frequency within a population (Keim et al., 2004). VNTRs that contained higher numbers of alleles tended to have higher diversity values (Table 2) and were more variable (Chiou et al., 2009). Four loci (Sty2, Sty3, Sty39 and Sty42) had low diversity values. The allelic diversity of all VNTRs, with the exception of Sty2, Sty3 and Sty42, were previously evaluated using 73 global $S$. Typhi isolates (Octavia \& Lan,
2009) and the diversity levels observed for the VNTRs were similar to those described in this study.

\section{Discriminatory power of MLVA and PFGE}

The DIs of various VNTR combinations (corresponding to different multilocus VNTR analyses) and PFGE methods were compared using a panel of 125 isolates. MLVA4, an assay based on analysis of the four most variable VNTRs (Sty25, Sty37, Sty41 and Sty45), displayed a higher DI than PFGE-XbaI, PFGE-BlnI and a combination of PFGE-XbaI and PFGE-BlnI for the $125 \mathrm{~S}$. Typhi isolates characterized (Table 3). MLVA8, including an additional four VNTR markers (Sty20, Sty40, Sty42 and Sty44), provided further discrimination over MLVA4 and displayed a significantly higher DI than the combination of PFGE-XbaI and PFGEBlnI. MLVA11, which includes three VNTRs (Sty2, Sty3 and Sty39) with low-variability in addition to the eight VNTRs of MLVA8, provided no additional discrimination over MLVA8. The combination of MLVA4 with either PFGE-XbaI or PFGE-BlnI yielded assays with a higher level of discrimination than the three methods alone. However, an assay using a combination of MLVA4 and both PFGE methods displayed the same level of discrimination as that using MLVA4 with just one of the PFGE methods. Similarly, the combination of MLVA8 with either PFGE$X b a \mathrm{I}$ or PFGE-BlnI displayed a higher level of discrimination than using any of these methods alone and the combination of MLVA8 with either one of the PFGE methods displayed the same level of discrimination as that obtained from MLVA8 or MLVA11 combined with both of the PFGE methods.

Based on the analyses of a small set of highly variable loci, MLVA is sufficient to replace PFGE in resolving closely 
Table 2. Characteristics of the 11 VNTRs observed in 125 Salmonella enterica serovar Typhi isolates and the primers used for their amplification

\begin{tabular}{|c|c|c|c|c|c|c|}
\hline VNTR locus (alias)* & $\begin{array}{l}\text { Size of repeat } \\
\text { unit }(b p)\end{array}$ & $\begin{array}{l}\text { PCR amplicon size } \\
\text { range (bp) }\end{array}$ & No. alleles & $\begin{array}{l}\text { Range of repeat } \\
\text { units }\end{array}$ & Allelic diversity & Primers $\left(5^{\prime}-3^{\prime}\right) \ddagger$ \\
\hline Sty2 & 6 & $219-225$ & 2 & $2-3$ & 0.02 & $\begin{array}{l}\text { Forward: VIC-TCGGCCAACGATAAGGATAC } \\
\text { Reverse: GTGGGCTATCACCGTTGTTC }\end{array}$ \\
\hline Sty20 (TR4500) & 6 & $173-240$ & 6 & $1-11(173) \dagger$ & 0.70 & $\begin{array}{l}\text { Forward: NED-CTGGCCCATATTGTGGAGAT } \\
\text { Reverse: AAGGTTCGTAAAGCCGTTGA }\end{array}$ \\
\hline Sty25 (TR4699) & 6 & $108-276$ & 25 & $4-32$ & 0.92 & $\begin{array}{l}\text { Forward: NED-CGGGCAATTCGAGATAGGTA } \\
\text { Reverse: TAGTTTTGGACCTGCCCATT }\end{array}$ \\
\hline Sty39 (Sal06) & 6 & $174-186$ & 3 & $5-7$ & 0.15 & $\begin{array}{l}\text { Forward: 6FAM-TTGGTCGCGGAACTATAACTG } \\
\text { Reverse: CTTCGTCTGATTGCCACTCC }\end{array}$ \\
\hline Sty40 (Sal20) & 3 & $224-266$ & 12 & $3-17$ & 0.82 & $\begin{array}{l}\text { Forward: VIC-AATCCGAATCAGCTCACCAG } \\
\text { Reverse: GTTTGCGGCTGTTCGTTTAC }\end{array}$ \\
\hline Sty41 (TR1 or Sal11) & 7 & $190-274$ & 13 & 5-17 & 0.90 & $\begin{array}{l}\text { Forward: PET-GCCAACGATCGCTACTTTTT } \\
\text { Reverse: GCGCATACTACACCGATCAC }\end{array}$ \\
\hline
\end{tabular}

${ }^{*}$ Alias loci, in parentheses, have been previously described: TR1, TR2 and TR5 (Liu et al., 2003); STTR5 (Lindstedt et al., 2003); Sal02, Sal06 and Sal20 (Ramisse et al., 2004 ); TR4500 and TR4699 (Octavia \& Lan, 2009).

$\dagger$ The range of repeat units for allele 173 of Sty20, which has a 7 bp deletion at the $5^{\prime}$-flanking region immediately adjacent to the repeats, is reported instead of that for the fragment size (bp). ¥Multiplex PCRs were performed using primers for the following VNTR loci: M1 (Sty20, Sty37, Sty39 and Sty40); M2 (Sty25, Sty41, Sty44 and Sty45); M3 (Sty2, Sty3 and Sty42). 
Table 3. Discriminatory index for the PFGE and MLVA methods based on the analysis of various numbers of VNTR markers

\begin{tabular}{|lccc|}
\hline Methods & No. genotypes & DI & 95 \% CI \\
\hline PFGE-XbaI & 71 & 0.980 & $0.971-0.989$ \\
PFGE-BlnI & 74 & 0.982 & $0.973-0.990$ \\
PFGE-XbaI + PFGE-BlnI & 84 & 0.986 & $0.979-0.993$ \\
MLVA4 & 106 & 0.993 & $0.986-1.000$ \\
MLVA8 & 109 & 0.996 & $0.993-0.999$ \\
MLVA11 & 109 & 0.996 & $0.993-0.999$ \\
MLVA4+PFGE-XbaI & 110 & 0.996 & $0.992-1.000$ \\
MLVA4+PFGE-BlnI & 110 & 0.996 & $0.992-1.000$ \\
MLVA4+PFGE-XbaI + PFGE-BlnI & 110 & 0.996 & $0.992-1.000$ \\
MLVA8 + PFGE-XbaI & 113 & 0.998 & $0.996-1.000$ \\
MLVA8 + PFGE-BlnI & 113 & 0.998 & $0.996-1.000$ \\
MLVA8 + PFGE-XbaI + PFGE-BlnI & 113 & 0.998 & $0.996-1.000$ \\
MLVA11 + PFGE-XbaI + PFGE-BlnI & 113 & 0.998 & $0.996-1.000$ \\
\hline
\end{tabular}

related isolates of some monomorphic bacteria for the investigation of disease outbreaks (Chiou et al., 2010; Noller et al., 2003; Torpdahl et al., 2007). A previous study (Liang et al., 2007) showed that MLVA, based on only four highly variable VNTR markers, exhibited a level of discrimination that was significantly higher than that provided by PFGE for the $S$. sonnei isolates analysed. The comparisons in this study also indicate that MLVA, based on the analysis of only four highly variable VNTR markers, has a higher DI for distinguishing between $S$. Typhi isolates than PFGE with one enzyme or even PFGE with two enzymes.

\section{Analysis of isolates indistinguishable by PFGE with two enzymes}

Of the 125 isolates, 57 in 16 clusters (C1-C16) could not be discriminated by PFGE with two enzymes, XbaI and BlnI (Table 1). Each of the PFGE clusters contained between two and nine isolates. Ten of the 16 clusters were epidemiologically unlinked. Six clusters (C4, C5, C12, C13, C14 and $\mathrm{C} 15)$ were epidemiologically related and had been recovered from patients in the same family or from close relatives (Epi1 and Epi-2), or from patients from the same area within a short time period (Epi-3, Epi-4 and Epi-5) (Table 1). One Epi-3 isolate had a different PFGE type from the other Epi-3 isolates in cluster $\mathrm{C} 12$ but shared an indistinguishable PFGE type with the C10 isolates. Three Epi-4 isolates had different PFGE types from other Epi-4 isolates in clusters C13 and C14 and one Epi-5 isolate had a different PFGE type from the other Epi-5 isolates. Epidemiologically unlinked isolates in some of the clusters were recovered over a long period of time. For example, the six isolates in the C2 cluster were recovered during 1998-2006 (Table 1).

The 57 isolates were separated into 40 MLVA4, 43 MLVA8 and 43 MLVA11 types. MLVA8 and MLVA11 displayed a high level of discrimination between the isolates with no epidemiological link. All but two pairs of the epidemiologically unlinked isolates could be distinguished by using
MLVA8 and MLVA11. Two epidemiologically unlinked isolates (NK07.052 and 04-139907) in cluster C9 shared the same MLVA profile (M.040) and two isolates (N05.0014 and C06.2070) in cluster C10 shared a common MLVA type (M.027) (Table 1). Isolates in each of the epidemiologically related clusters C4, C5 and C15 (belonging to Epi1, Epi-2 and Epi-5, respectively) shared a common MLVA type. Three MLVA types were identified in the three C12 isolates, three MLVA types were identified in the four C13 isolates and two MLVA types were identified in the seven C14 isolates.

In the 16 clusters, variation occurred in eight VNTRs at the following frequencies: Sty45 (in 9 clusters), Sty25 (8), Sty37 (8), Sty41 (6), Sty44 (6), Sty20 (5), Sty40 (5) and Sty42 (1). The variation frequency correlated with the number of alleles $\left(r^{2}=0.9259\right)$ and the allelic diversity $\left(r^{2}=0.9674\right)$ for the 11 VNTRs in the panel of 125 S. Typhi isolates. Accordingly, VNTRs which are more diverse in S. Typhi populations are more variable.

The data showed that the PFGE-XbaI and PFGE-BlnI methods as well as a combination of these methods were insufficient in distinguishing between many epidemiologically unlinked isolates, even though their isolation dates were 8 years apart. Isolates in 10 of the 16 clusters were epidemiologically unlinked but could not be distinguished from one another by the two PFGE methods. In contrast, all but two pairs of epidemiologically unlinked isolates from the 10 clusters were discriminated by MLVA8 and MLVA11.

\section{Genetic relationships and epidemiology}

The genetic relationships among the 125 isolates were determined based on the MLVA11 profiles by using the MST algorithm. An identical phylogeny was obtained using MLVA8 profiles because this method displayed the same level of discrimination as MLVA11. As shown in the MST tree (Fig. 1), isolates from each of the five epidemiological clusters (Epi-1-Epi-5) shared either a common MLVA 


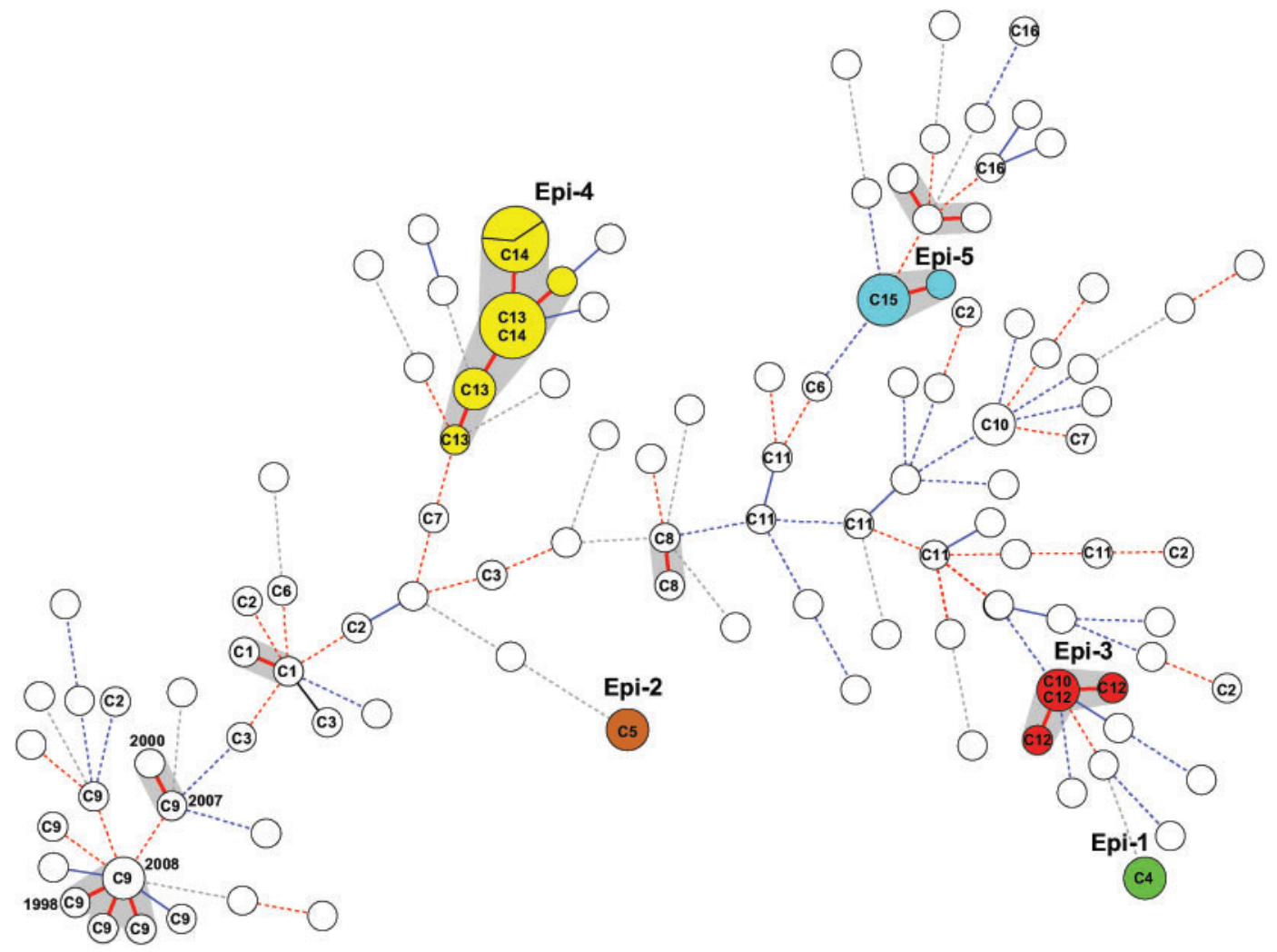

Fig. 1. Genetic relationships among 125 isolates based on MLVA11 profiles using the MST tree algorithm. The area of the circle is proportional to the number of isolates belonging to the MLVA type. MLVA types for the isolates in the 16 PFGE clusters $(\mathrm{C} 1-\mathrm{C} 16)$ are indicated inside the circles. A distance of one locus between two MLVA types is indicated by a thick red line, a distance of two loci by a thin blue line, a distance of three loci by a broken red line and a distance of four loci by a broken blue line. Distances of five loci or greater are marked by a broken grey line. MLVA types differing at one locus are marked with a grey shadow. The MLVA types for isolates in the five epidemiologically related clusters (Epi-1-Epi-5) are marked in different colours. The years of isolation are indicated for two pairs of isolates that emerged 7 and 10 years apart.

profile or close MLVA profiles. The isolates from clusters Epi-1 and Epi-2 shared a common MLVA profile. Multiple MLVA profiles were found in the isolates in clusters Epi-3, Epi-4 and Epi-5 but they were closely linked. The four isolates from cluster Epi-3 possessed three MLVA profiles that differed at Sty 25 with 18, 19 and 20 repeat units. Epi-4 contained 14 isolates that emerged in Northern Taiwan from June 19 to July 21, 2005. These isolates could be divided into five types using PFGE with the two enzymes and five MLVA types that differed at Sty25 (with 14 and 15 repeat units), Sty 42 (with 3 and 4 repeat units), Sty 44 (with 6 and 7 repeat units) and Sty 45 (with 18 and 19 repeat units). The four Epi-5 isolates differed at Sty 45 with 37 or 38 repeat units.

Typhoid fever is an uncommon disease in Taiwan. In total, 703 cases were confirmed in 1996-2009 with a mean annual incidence rate of 0.22 cases per 10000 people in the population. At least $35 \%$ of the cases were imported. It was quite unusual that a cluster of 14 cases involving Epi-4 isolates emerged within a county in a 1-month period. These cases should have had a common source of infection but this was in doubt because multiple PFGE and MLVA types were identified in the isolates. Recently, we detected two MLVA types with a difference of three VNTRs in isolates obtained from a long-term carrier, indicating that the bacteria had evolved into multiple genotypes in the years of carriage. This suggests that Epi- 4 isolates with multiple genotypes could come from a common source, a long-term carrier.

Among the epidemiologically unlinked PFGE clusters, two isolates in C10 (recovered on July 4, 2005 and November 6, 2006) as well as two isolates in C9 (recovered on August 15, 2007 and May 19, 2008) shared an identical MLVA type. MLVA types for members of clusters C1, C8 and C9 were closely linked; the MLVA types for members of $\mathrm{C} 1$ and $\mathrm{C} 8$ had one-locus difference and those for C9 isolates differed by up to three loci between two closest types. For two pairs of isolates that emerged 7 and 10 years apart, their MLVA profiles differed only at one locus (Fig. 1). In contrast, members of clusters C2, C3, C6, C7, C11 and C16 had significantly diversified. The isolates in each of the clusters differed in 4-7 of their VNTRs. The MLVA types for 
isolates in clusters C2 and C16 were the most diverse, differing at seven loci.

Isolates that shared indistinguishable PFGE patterns should have closer genetic relationships than those with different PFGE patterns; however, there were distantly linked isolates in PFGE clusters C2, C3, C6, C7, C11 and C16 as shown in the MST based on MLVA11 profiles (Fig. 1). VNTR markers have a wide range of evolutionary rates; therefore, they can be used to investigate genetic relationships among isolates that have evolved over different timescales (Chiou et al., 2009; Pourcel et al., 2004; Wang et al., 2009). Rapidly evolving VNTRs are suitable for investigating genetic relationships among closely related isolates in short-term epidemiological surveillance studies, whereas slowly evolving VNTRs are more useful for establishing clearer clonal relationships among isolates in the investigation of their long-term epidemiology, such as the global transmission of important clones (Chiou et al., 2009). Because seven of the 11 VNTRs for $S$. Typhi were highly variable, the panel of 11 VNTRs is only useful in establishing accurate genetic relationships among very closely related $S$. Typhi isolates, such as Epi-1-Epi-5 isolates.

\section{ACKNOWLEDGEMENTS}

This work was supported by the Centers for Disease Control, Department of Health of Taiwan (grant no. DOH98-DC-2022).

\section{REFERENCES}

Boxrud, D., Pederson-Gulrud, K., Wotton, J., Medus, C., Lyszkowicz, E., Besser, J. \& Bartkus, J. M. (2007). Comparison of multiple-locus variable-number tandem repeat analysis, pulsed-field gel electrophoresis, and phage typing for subtype analysis of Salmonella enterica serotype Enteritidis. J Clin Microbiol 45, 536-543.

Caugant, D. A., Frøholm, L. O., Bøvre, K., Holten, E., Frasch, C. E., Mocca, L. F., Zollinger, W. D. \& Selander, R. K. (1986). Intercontinental spread of a genetically distinctive complex of clones of Neisseria meningitidis causing epidemic disease. Proc Natl Acad Sci U S A 83, 4927-4931.

Chang, C. H., Chang, Y. C., Underwood, A., Chiou, C. S. \& Kao, C. Y. (2007). VNTRDB: a bacterial variable number tandem repeat locus database. Nucleic Acids Res 35 (Database issue), D416-D421.

Chiou, C. S., Liao, J. C., Liao, T. L., Li, C. C., Chou, C. Y., Chang, H. L., Yao, S. M. \& Lee, Y. S. (2006). Molecular epidemiology and emergence of worldwide epidemic clones of Neisseria meningitidis in Taiwan. BMC Infect Dis 6, 25.

Chiou, C. S., Watanabe, H., Wang, Y. W., Wang, W. L., Terajima, J., Thong, K. L., Phung, D. C. \& Tung, S. K. (2009). Utility of multilocus variable-number tandem-repeat analysis as a molecular tool for phylogenetic analysis of Shigella sonnei. J Clin Microbiol 47, 11491154.

Chiou, C. S., Hung, C. S., Torpdahl, M., Watanabe, H., Tung, S. K., Terajima, J., Liang, S. Y. \& Wang, Y. W. (2010). Development and evaluation of multilocus variable number tandem repeat analysis for fine typing and phylogenetic analysis of Salmonella enterica serovar Typhimurium. Int J Food Microbiol 142, 67-73.

Connor, B. A. \& Schwartz, E. (2005). Typhoid and paratyphoid fever in travellers. Lancet Infect Dis 5, 623-628.
Crump, J. A., Luby, S. P. \& Mintz, E. D. (2004). The global burden of typhoid fever. Bull World Health Organ 82, 346-353.

Grundmann, H., Hori, S. \& Tanner, G. (2001). Determining confidence intervals when measuring genetic diversity and the discriminatory abilities of typing methods for microorganisms. J Clin Microbiol 39, 4190-4192.

Hunter, P. R. (1990). Reproducibility and indices of discriminatory power of microbial typing methods. J Clin Microbiol 28, 1903-1905.

Hyytiä-Trees, E. K., Cooper, K., Ribot, E. M. \& Gerner-Smidt, P. (2007). Recent developments and future prospects in subtyping of foodborne bacterial pathogens. Future Microbiol 2, 175-185.

Keim, P., Van Ert, M. N., Pearson, T., Vogler, A. J., Huynh, L. Y. \& Wagner, D. M. (2004). Anthrax molecular epidemiology and forensics: using the appropriate marker for different evolutionary scales. Infect Genet Evol 4, 205-213.

Kidgell, C., Reichard, U., Wain, J., Linz, B., Torpdahl, M., Dougan, G. \& Achtman, M. (2002). Salmonella typhi, the causative agent of typhoid fever, is approximately 50,000 years old. Infect Genet Evol 2, 39-45.

Liang, S. Y., Watanabe, H., Terajima, J., Li, C. C., Liao, J. C., Tung, S. K. \& Chiou, C. S. (2007). Multilocus variable-number tandem-repeat analysis for molecular typing of Shigella sonnei. J Clin Microbiol 45, 3574-3580.

Liao, J. C., Li, C. C. \& Chiou, C. S. (2006). Use of a multilocus variablenumber tandem repeat analysis method for molecular subtyping and phylogenetic analysis of Neisseria meningitidis isolates. BMC Microbiol 6, 44 .

Lindstedt, B. A., Heir, E., Gjernes, E. \& Kapperud, G. (2003). DNA fingerprinting of Salmonella enterica subsp. enterica serovar typhimurium with emphasis on phage type DT104 based on variable number of tandem repeat loci. J Clin Microbiol 41, 1469-1479.

Liu, Y., Lee, M. A., Ooi, E. E., Mavis, Y., Tan, A. L. \& Quek, H. H. (2003). Molecular typing of Salmonella enterica serovar Typhi isolates from various countries in Asia by a multiplex PCR assay on variable-number tandem repeats. J Clin Microbiol 41, 4388-4394.

Maiden, M. C., Bygraves, J. A., Feil, E., Morelli, G., Russell, J. E., Urwin, R., Zhang, Q., Zhou, J., Zurth, K. \& other authors (1998). Multilocus sequence typing: a portable approach to the identification of clones within populations of pathogenic microorganisms. Proc Natl Acad Sci U S A 95, 3140-3145.

Marsh, J. W., O'Leary, M. M., Shutt, K. A., Pasculle, A. W., Johnson, S., Gerding, D. N., Muto, C. A. \& Harrison, L. H. (2006). Multilocus variable-number tandem-repeat analysis for investigation of Clostridium difficile transmission in hospitals. J Clin Microbiol 44, 2558-2566.

McClelland, M., Sanderson, K. E., Clifton, S. W., Latreille, P., Porwollik, S., Sabo, A., Meyer, R., Bieri, T., Ozersky, P. \& other authors (2004). Comparison of genome degradation in Paratyphi A and Typhi, human-restricted serovars of Salmonella enterica that cause typhoid. Nat Genet 36, 1268-1274.

Noller, A. C., McEllistrem, M. C., Pacheco, A. G., Boxrud, D. J. \& Harrison, L. H. (2003). Multilocus variable-number tandem repeat analysis distinguishes outbreak and sporadic Escherichia coli O157: H7 isolates. J Clin Microbiol 41, 5389-5397.

Octavia, S. \& Lan, R. (2007). Single-nucleotide-polymorphism typing and genetic relationships of Salmonella enterica serovar Typhi isolates. J Clin Microbiol 45, 3795-3801.

Octavia, S. \& Lan, R. (2009). Multiple-locus variable-number tandemrepeat analysis of Salmonella enterica serovar Typhi. J Clin Microbiol 47, 2369-2376.

Pourcel, C., André-Mazeaud, F., Neubauer, H., Ramisse, F. \& Vergnaud, G. (2004). Tandem repeats analysis for the high resolution phylogenetic analysis of Yersinia pestis. BMC Microbiol 4, 22. 
Ramisse, V., Houssu, P., Hernandez, E., Denoeud, F., Hilaire, V., Lisanti, O., Ramisse, F., Cavallo, J. D. \& Vergnaud, G. (2004). Variable number of tandem repeats in Salmonella enterica subsp. enterica for typing purposes. J Clin Microbiol 42, 5722-5730.

Ribot, E. M., Fair, M. A., Gautom, R., Cameron, D. N., Hunter, S. B., Swaminathan, B. \& Barrett, T. J. (2006). Standardization of pulsedfield gel electrophoresis protocols for the subtyping of Escherichia coli O157:H7, Salmonella, and Shigella for PulseNet. Foodborne Pathog Dis 3, 59-67.

Roumagnac, P., Weill, F. X., Dolecek, C., Baker, S., Brisse, S., Chinh, N. T., Le, T. A., Acosta, C. J., Farrar, J. \& other authors (2006). Evolutionary history of Salmonella typhi. Science 314, 1301-1304.

Spratt, B. G. (2004). Exploring the concept of clonality in bacteria. Methods Mol Biol 266, 323-352.

Swaminathan, B., Gerner-Smidt, P., Ng, L. K., Lukinmaa, S., Kam, K. M., Rolando, S., Gutiérrez, E. P. \& Binsztein, N. (2006). Building
PulseNet International: an interconnected system of laboratory networks to facilitate timely public health recognition and response to foodborne disease outbreaks and emerging foodborne diseases. Foodborne Pathog Dis 3, 36-50.

Tien, Y. Y., Wang, Y. W., Tung, S. K., Liang, S. Y. \& Chiou, C. S. (2011). Comparison of multilocus variable-number tandem repeat analysis and pulsed-field gel electrophoresis in molecular subtyping of Salmonella enterica serovars Paratyphi A. Diagn Microbiol Infect Dis 69, 1-6.

Torpdahl, M., Sørensen, G., Lindstedt, B. A. \& Nielsen, E. M. (2007). Tandem repeat analysis for surveillance of human Salmonella Typhimurium infections. Emerg Infect Dis 13, 388-395.

Wang, Y. W., Watanabe, H., Phung, D. C., Tung, S. K., Lee, Y. S., Terajima, J., Liang, S. Y. \& Chiou, C. S. (2009). Multilocus variablenumber tandem repeat analysis for molecular typing and phylogenetic analysis of Shigella flexneri. BMC Microbiol 9, 278. 